\title{
Superfuid-insulator transitions at non-integer filling in optical lattices of fermionic atoms
}

\author{
Chih-Chun Chien, Yan He, Qijin Chen, and K. Levin \\ James Franck Institute and Department of Physics, University of Chicago, Chicago, Illinois 60637
}

(Dated: November 20, 2018)

\begin{abstract}
We determine the superfluid transition temperatures $T_{c}$ and the ground states of the attractive Hubbard model and find new insulating phases associated with non-integer filling at sufficiently strong pairing attraction $|U|$. These states, distinct from band and Mott insulating phases, derive from pair localization; pair hopping at large $|U|$ and high densities is impeded by inter-site, inter-pair repulsive interactions. The best way to detect the breakdown of superfluidity is using fermionic optical lattices which should reveal new forms of "bosonic" order, reflecting ground state pairing without condensation.

PACS numbers: 03.75.Hh, 03.75.Ss, 74.20.-z
\end{abstract}

The attractive Hubbard Model has captured the attention [1] of the theoretical and experimental communities, although this model is far from fully understood. It is hoped that experiments using ultracold fermionic atoms in optical lattices [2] will shed light both on competing ground states and on finite temperature $T$ effects, and moreover, provide insights into high temperature superconductivity, largely through the very ubiquitous "pseudogap" effects. These derive from [3] the fact that as the attraction becomes stronger, pairing takes place at a different temperature, $T^{*}$ from superfluid condensation, $T_{c}$.

For bosonic systems at half filling the strong (repulsive) interaction limit gives rise to superfluid-Mott insulator transitions [4]. It is a central goal of this paper to investigate this counterpart strong (attractive) interaction regime for fermions and demonstrate that superfluid-insulator (SI) transitions (away from integer filling) are also present. Indeed, the latest experiments on fermions[2] reveal competing insulating and superfluid ground states. It is not yet clear whether these insulating phases are band insulators or localization-induced insulating phases. We note that the largest coupling regime we address in this paper has not yet studied by Monte Carlo [5] or other methods [6], despite the fact that a comparably strong (dimensionless) interaction strength is required for bosonic systems to undergo SI transitions.

Our finite $T$ approach to the Hubbard model includes pair fluctuations in a manner which is consistent with the standard BCS-Leggett ground state and, most importantly, with a proper and physical treatment of pseudogap effects. We stress, however, that these non-condensed pair effects must necessarily be treated differently from previous NozièresSchmitt-Rink [7] based approaches to $T \neq 0$. Indeed, following Ref. [7], essentially all other pairing fluctuation approaches to BCS-BEC crossover contain an inherent inconsistency; they presume that in the fermionic dispersion relation $E_{\mathbf{k}}=\sqrt{\xi_{\mathbf{k}}^{2}+\Delta^{2}(T)}$, the so-called pairing gap $\Delta$ vanishes at and above $T_{c}$. These non-condensed pairs (present for all $0<T<T^{*}$ ) are also essential for arriving at physical values for the transition temperature $T_{c}$. Without including them it is not possible to know about ground states with $T_{c}=0$, which will naturally occur, e.g., in the present theory [8] in twodimensional lattices, as compatible with the Mermin-Wagner theorem (in the absence of Kosterlitz-Thouless or other topo- logical order). This underlines the fact that one cannot solve the BCS-Leggett $T=0$ equations in isolation to characterize the stable ground states.

Our principal result is that, in addition to the expected band insulating state (at filling $n=2$ ), a new insulating phase, which is stabilized by pair fluctuations, emerges when $n$ and the interaction strength exceed appropriate critical values. Strikingly, the critical value for the dimensionless interaction strength is comparable to that found for the Bose Hubbard model [4]. This new insulating phase is different from the Mott or band insulator, both of which occur only at integer filling, but like the Mott case it is due to localization (of fermion pairs). To address cold atom optical lattices, we also extend the attractive Hubbard model to a two-channel model and find that the insulating phases at non-integer filling survive; thus, pair localization is a robust effect.

Our work can be contrasted with previous studies, which focused only on $T=0[9,10,11,12]$ and with Monte Carlo (MC) [5] as well as dynamical mean field theoretic approaches [6]. In the moderate coupling regime, where comparisons can be made, we find transition temperatures to be of comparable order of magnitude, albeit slightly larger (factors of 2). In part this derives from the fact that the standard BCS-Leggett ground state considered here does not include [6] the GorkovMelik-Barkhudarov [13] effect. This semi-quantitative difference should not be of concern because different MC calculations [14, 15] of $T_{c}$ in a unitary Fermi gas have not yet converged to better than factors of 1.5 .

Of particular interest here are the properties of the attractive Hubbard model, in the limit that the attraction $|U|$ is much larger than the hopping $t$. While this model exhibits a smooth crossover from BCS to Bose-Einstein condensation (BEC) with increasing $|U|$, it should be stressed that the BEC here is very different from that of true bosons, since the hopping of the pairs involves, as an intermediate or virtual step, their unbinding into fermions. In this way the inverse pair mass was argued [1, 7] to vary as $t^{2} /|U|$. Important for the present purposes is the fact that there is also a nearest neighbor repulsion [1, 7] which is of the same magnitude as the effective pair hopping term. On this basis it has been argued that [1] "at higher densities the overlap of the bound pairs severely restricts their motion". This quotation is particularly relevant to our findings; we show that at sufficiently high $n$ the pair motion is 
so restricted that their effective mass diverges prematurely before $|U| \rightarrow \infty$. This localization, in turn, is associated with the breakdown of superfluidity.

This frustration of superfluidity at high $n$ can also be addressed via a mapping to a magnetic model. When $|U| / t \gg 1$ the attractive Hubbard model is equivalent to [1] an effective quantum XXZ model with coupling constants $J_{X X}=-J_{Z}$, both of which are proportional to $t^{2} /|U|$. This mapped problem is subject to the constraint that the average magnetization along the $z$-axis is fixed at $(n-1) / 2$, making this a rather difficult magnetic model to solve in general. Superfluidity corresponds to ordering in the $x-y$ plane. When $n$ is small, the constraint is straightforward to implement and superfluidity emerges. However, at high densities $(n \approx 1)$ new states with order along the $z$-axis are expected [1] to emerge, thereby, destroying the superfluid phases. Indeed, it is well known that for $n$ strictly equal to 1 , the simple superfluid ground state is undermined as a result of a degeneracy with a charge ordered ground state [1].

We now address these effects by investigating the physics of fermions which interact via $s$-wave attraction in a three dimensional (3D) square lattice, we start with the one-channel attractive Hubbard model Hamiltonian

$$
\begin{aligned}
H_{f}= & \sum_{\mathbf{k} \sigma} \xi_{\mathbf{k}} c_{\mathbf{k} \sigma}^{\dagger} c_{\mathbf{k} \sigma} \\
& +U \sum_{\mathbf{k k}^{\prime} \mathbf{q}} c_{\mathbf{k}+\mathbf{q} / 2 \uparrow}^{\dagger} c_{-\mathbf{k}+\mathbf{q} / 2 \downarrow}^{\dagger} c_{-\mathbf{k}^{\prime}+\mathbf{q} / 2 \downarrow} c_{\mathbf{k}^{\prime}+\mathbf{q} / 2 \uparrow} \cdot(1)
\end{aligned}
$$

Here $\xi_{\mathbf{k}}=\epsilon_{\mathbf{k}}-\mu$, where $\mu$ is the chemical potential. The oneparticle energy dispersion is $\epsilon_{\mathbf{k}}=2 t\left[3-\cos k_{x}-\cos k_{y}-\right.$ $\left.\cos k_{z}\right]$ in a one-band nearest-neighbor tight-binding approximation, where the values of $\mathbf{k}$ are restricted in the first Brillouin zone, and we set lattice constant $a_{0}=1$. $U$ represents the attractive on-site coupling. "Resonant" scattering, which corresponds to an infinite two body scattering length, occurs at $U / t \approx-7.915$, in agreement with Ref. [16]. Note that by adopting the Hubbard model we drop any terms associated with direct pair hopping which should not be important in the regime we focus on here where $\mu$ is positive or only slightly negative. We consider a one band model, on the premise that multi-band effects will change the results quantitatively but not qualitatively. There is still uncertainty in the literature [12] about whether or not an effective one band [9] model is adequate.

We use a $T$-matrix formalism [3, 17, 18] to address finite temperature. This particular $T$-matrix approach has a crucial advantage because it leads to physical results for the superfluid density $n_{s}(T)$. This single valued, monotonic and continuous behavior (from zero to $T_{c}$ ) for $n_{s}(T)$ is not found in other theories; this physical behavior can be traced to a self consistent treatment of pseudogap effects [3], in which pair fluctuations enter into both the gap and the number equations in a fully self-consistent fashion

Details of this formalism can be found in [3]. We define the noncondensed pair propagator as $t_{p g}(Q)=U /[1+U \chi(Q)]$, where, as in Ref. [19], our choice for the pair susceptibility, given by $\chi(Q)=\sum_{K} G_{0}(Q-K) G(K)$, can be derived from decoupling the Green's function equations of motion.
Here $G(K)$ and $G_{0}(K)=i \omega_{n}-\xi_{\mathbf{k}}$ are the full and bare Green's functions. $K \equiv\left(i \omega_{n}, \mathbf{k}\right)$ and $Q \equiv\left(i \Omega_{m}, \mathbf{q}\right)$ are fourvectors with $\sum_{K} \equiv T \sum_{\omega_{n}} \sum_{\mathbf{k}}$. Below $T_{c}$, the self-energy $\Sigma(K)=\sum_{Q} t(Q) G_{0}(Q-K)$ can be well approximated by the BCS form, $\Sigma(K)=-\Delta^{2} G_{0}(-K)$, where $t(Q)=$ $-\left(\Delta_{s c}^{2} / T\right) \delta(Q)+t_{p g}(Q)$, and $\Delta_{s c}$ is the superfluid order parameter. In the superfluid state, the "gap equation" is given by the pairing instability condition $t_{p g}^{-1}(0)=U^{-1}+\chi(0)=0$, which is equivalent to the BEC condition on the pairs. Therefore, we have

$$
\frac{1}{U}=-\sum_{\mathbf{k}}\left[\frac{1-2 f\left(E_{\mathbf{k}}\right)}{E_{\mathbf{k}}}\right] .
$$

Here $f(x)$ is the Fermi distribution function. Similarly, the average [20] density $n$ in a lattice, derived from $n=$ $\sum_{K, \sigma} G_{\sigma}(K)$, is given by

$$
n=\sum_{\mathbf{k}}\left[\left(1-\frac{\xi_{k}}{E_{k}}\right)+2 f\left(E_{k}\right)\left(\frac{\xi_{k}}{E_{k}}\right)\right] .
$$

We next determine the dispersion relation and the number density for noncondensed pairs. From the self-energy expression one obtains $\Delta^{2}=\Delta_{c r}^{2}+\Delta_{p g}^{2}$ where the pseudogap contribution satisfies [3, 18, 19]

$$
\Delta_{p g}^{2} \equiv-\sum_{Q} t_{p g}(Q)
$$

which can be shown to vanish at $T=0$. The critical temperature $T_{c}$ is defined as the lowest temperature where $\Delta_{s c}=0$. In the superfluid phase (pair chemical potential $\mu_{\text {pair }}=0$ ), and at small $Q, t_{p g}^{-1}(Q)=\chi(Q)-\chi(0) \approx Z_{1} \Omega^{2}+\left|Z_{0}\right| \Omega-\xi^{2} q^{2}$. Except when particle-hole symmetry is present, e.g., at very weak coupling and near half filling, we find $Z_{1} \ll\left|Z_{0}\right|$, which thus is irrelevant. Near $n=1$, the $Z_{1} \Omega^{2}$ term enters and regularizes the van Hove singularity. To first order in $\Omega$ one can write $\Delta_{p g}^{2}=\left|Z_{0}\right|^{-1} \sum_{\mathbf{q}} b\left(\Omega_{\mathbf{q}}\right)$, where $b(x)$ is the Bose distribution function. At sufficiently low $\mathbf{q}$, we can approximate the dispersion of the noncondensed pairs by $\Omega_{q}=q^{2} / 2 M^{*}$, where $M^{*}$ is the effective mass of pairs at the bottom of the band.

In Fig. 11 we show $T_{c} / E_{F}$ as a function of $U / t$ for several values of $n$ in the one-channel model. Here $E_{F}$ is the Fermi energy of a non-interacting Fermi gas with the same filling factor. As in $\mathrm{MC}$ simulations, $T_{c}$ exhibits a maximum near resonance and decreases slightly when $U / t$ is away from resonance. When $n$ is small, we find a long tail in $T_{c}$ proportional to $t^{2} /|U|$, as expected [7]. Importantly, it is observed that when $n>n_{c} \approx 0.53, T_{c}$ vanishes provided $U / t$ exceeds a critical value $(U / t)_{c}$. It should be noted that at the insulating onset point, we find $\mu$ is zero or slightly positive, far from the true bosonic regime. The inclusion of pair fluctuation effects is essential here for establishing $T_{c}=0$ beyond $(U / t)_{c}$. This result can be contrasted with the predicted ground state of strict mean-field theory shown in the inset of Fig 1 where $\Delta / t$ vanishes only at $n=0$ and $n=2$ (band insulator).

In Fig. 2 we show typical phase diagrams at low and high densities. In contrast to $T_{c}$, the pairing onset temperature $T^{*}$ 


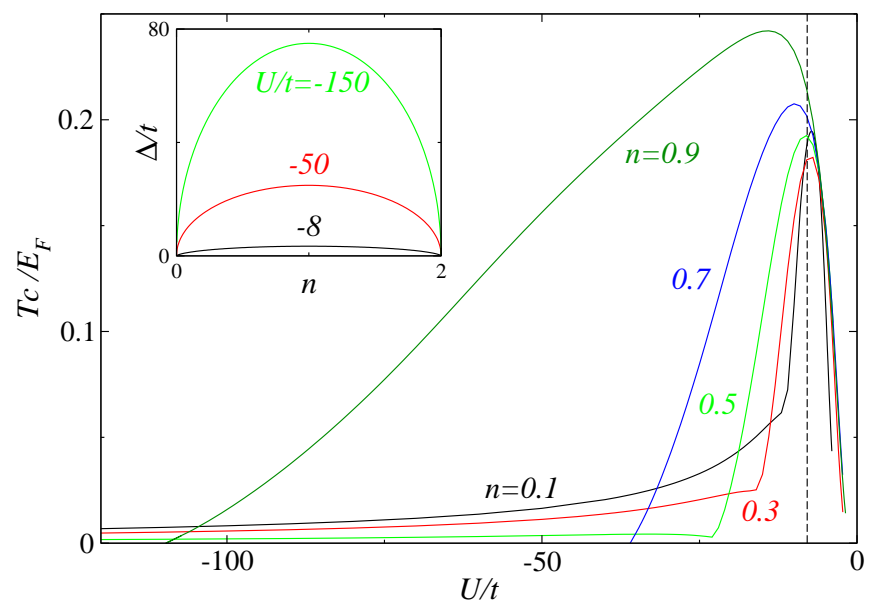

Figure 1: $T_{c} / E_{F}$ as a function of $U / t$. The corresponding value of $n$ is marked next to each curve. The vertical dashed line indicates the unitary limit. Inset: $T=0$ mean-field results for $\Delta / t$ as a function of $n$.

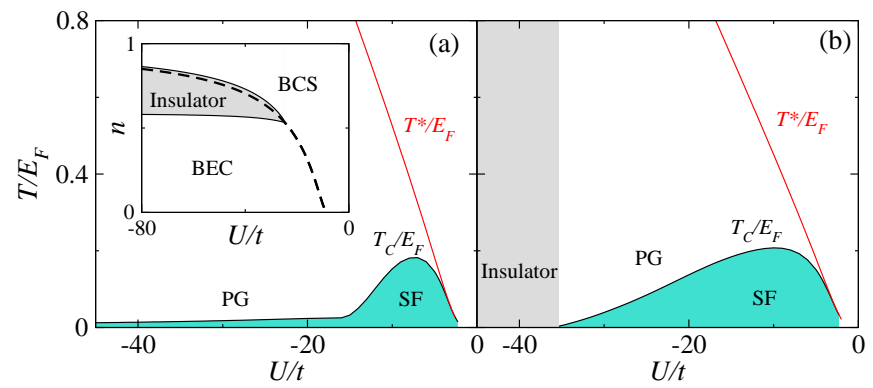

Figure 2: Phase diagrams of one-channel attractive Hubbard model at (a) $n=0.3$ and (b) $n=0.7$. 'SF' denotes superfluid, 'PG' denotes pseudogap phase and 'Insulator' in (b) schematically indicates breakdown of ground state superfluidity. Inset: $T=0$ phase diagram. Here the boundary separating BCS and BEC regimes is determined when $\mu$ reaches the bottom of the band. The gray shaded area shows where superfluid does not exist, corresponding to the 'Insulator' regime.

(as estimated from the strict mean-field solution for $T_{c}$ ), increases monotonically with $|U| / t$. The high density phase diagram, in particular, bears some similarity to its counterpart in high temperature superconductors [3]. In both figures, as in high $T_{c}$ superconductors, we see an anti-correlation between the behavior of $T_{c}$ and $T^{*}$, associated with lattice [1] as well as pseudgogap effects [8]. The complete suppression of superfluidity in Fig. 2(b) can be shown explicitly to come from localization of pairs; that is, the effective pair mass $M^{*} \rightarrow \infty$.

In the inset of Fig. 2 we show the $T=0$ phase diagram, in which "BCS" ("BEC") denote states with $\mu$ higher (lower) than the bottom of the band. The shaded regime corresponds to where there is a non-superfluid ground state. The lowest value of $(|U| / t)_{c} \approx 25$ occurs at $n=0.53$. This is comparable in magnitude to the critical value $|U| / t \approx 35$ of the Mott-superfluid transition of the $3 \mathrm{D}$ boson (repulsive) Hubbard model with filling factor 1 [4], although the physical origin of the two insulators is different.
In actual experiments, attractive interactions between the atoms are generated by Feshbach resonance effects, which in principle, require a two-channel description. Previous work on the two-channel model in a lattice concentrated on the superfluid-Mott transition in the strongly-interacting regime near $n=2[10,11]$. Other recent work discussed band insulators in the weakly-interacting regime [12]. The generalization of our $T$-matrix formalism to the twochannel model for Fermi gases was presented in [18]. To take into account the closed-channel molecules, the Hamiltonian is extended to $H=H_{f}+H_{b}+H_{f b}$, where $H_{b}=-\sum_{\mathbf{q}} E_{\mathbf{q}}^{m} b_{\mathbf{q}}^{\dagger} b_{\mathbf{q}}$ describes the hopping of molecules and $H_{f b}=\sum_{\mathbf{q}, \mathbf{k}} g\left(b_{\mathbf{q}}^{\dagger} c_{\mathbf{q} / 2-\mathbf{k} \downarrow} c_{\mathbf{q} / 2+\mathbf{k} \uparrow}+\right.$ h.c. $)$ describes conversion between molecules and fermion pairs. Here $E_{\mathbf{q}}^{m}=$ $\epsilon_{\mathbf{q}}^{m}-2 \mu+\nu, \nu$ is the magnetic detuning, and $g$ is the coupling constant for molecule-pair conversion. The strength of the attractive pairing interaction is modified relative to the one band case [18] to

$$
U_{e f f}(Q)=U+g^{2} D_{0}(Q)
$$

where $D_{0}(Q)=1 /\left(i \Omega_{n}-E_{\mathbf{q}}^{m}\right)$ is the propagator for noninteracting closed channel molecules. The gap equation involves only $U_{\text {eff }} \equiv U_{\text {eff }}(Q=0)$. The density in the lattice now becomes $n=n_{f}+2 n_{b}+2 n_{b}^{0}$ where the open-channel contribution $n_{f}$ is given by Eq. (3) and the closed-channel contribution comes from the molecular condensate $\left(2 n_{b}^{0}\right)$, and non-condensed molecules $\left(2 n_{b}\right)$. The energy dispersion of molecules has a Bloch-like dispersion, just as found for the fermions. In the long wavelength limit, we may expand this Bloch band dispersion as $\epsilon_{\mathbf{q}}^{m} \approx q^{2} / 2 M_{b}$, where $M_{b}=2 / t$ is the effective bare mass of the molecules. The fermion pairs have a similar $q^{2}$ dispersion and, in general, hybridize strongly with these closed-channel molecules.

In our Hamiltonian we have dropped a direct closedchannel boson-boson repulsion. This was included in previous work [10, 11] which aimed to create Mott insulating phases associated with the closed channel. At $n=2$, because the closed channel represents a band which is never completely filled (due to the presence of the open channel), we find that localized states in the strict Mott sense are not obtainable as is consistent with Ref. [11]. In this way we argue that it is appropriate here to drop the intra-closed-channel interactions. We note parenthetically that to obtain a Mott insulating state one possibility is to treat the closed channel as composite fermion pairs (or hard-core bosons [1]) and to consider filling above $n=2$.

Figure 3 shows $T_{c} / E_{F}$ in the two-channel model as a function of $U_{e f f} / t$ for [(a) and (c)] narrow and (b) broad Feshbach resonances. Here $U_{\text {eff }}$ is tuned via $\nu$ with fixed $U$ and $g$ in (a) and (b) and via $U$ with fixed $\nu$ and $g$ in (c). Figure 3 (a) corresponds to the case in which there is a considerable admixture of both closed and open channels. We take $U / t=-6$ and consider a relatively narrow resonance, $g / t=-60$, as well as two values of $n=0.7$ and $n=2$. For $n=0.7, T_{c}$ is first suppressed by the opening of the pseudogap as $-U_{\text {eff }}$ increases; then $T_{c}$ eventually increases with increasing $-U_{\text {eff }}$ as fermions are converted into closed-channel molecules which have a finite BEC transition temperature. For 


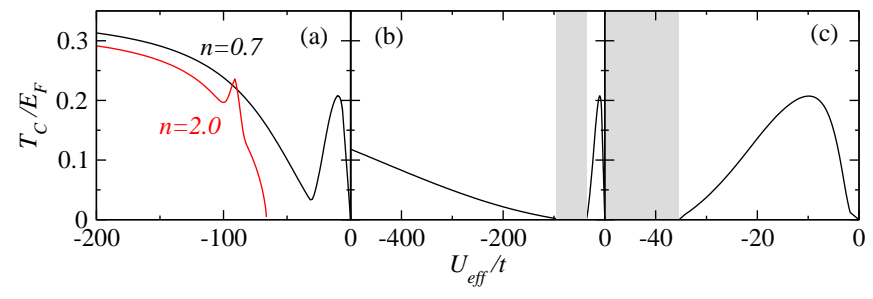

Figure 3: $T_{c} / E_{F}$ as a function of pairing interaction $U_{\text {eff }} / t$ in the two-channel model, as tuned by (a,b) detuning $\nu$ and (c) $U$ for relatively $(a, c)$ narrow and (b) broad Feshbach resonances. There parameters are: (a) $g / t=-60, U / t=-6, n=0.7$ (black) and 2 (red curve); (b) $n=0.7, g / t=-600, U / t=-6$; (c) $n=0.7$, $g / t=-60, \nu / t=500$. The shaded regimes in (b) and (c) schematically indicate the new, non-integer-filling insulating states.

$n=2$, the cusp in the $T_{c}$ curve comes from the van Hove singularity at $n_{f}=1$. For a large range of $U_{e f f}, T_{c}$ for $n=2$ is effectively zero [21], until a sufficient number of open channel pairs are converted to closed-channel molecules. Importantly, for this case, the insulating state thereby observed is a band insulator.

When we decrease the participation of closed channel molecules by either increasing $-U / t$ or $|g| / t$, insulating states (of the new form, at non-integer filling) start to emerge. In Fig. 3 b) we demonstrate these insulating states by plotting $T_{c} / E_{F}$ for $n=0.7$ at $U / t=-6$ and a large $|g| / t=600$ (wide resonance) as a function of $U_{\text {eff }} / t$ by tuning $\nu$. In Fig. 3] c) we plot the counterpart curve for a narrower resonance $(g / t=-60)$ as a function of $U_{\text {eff }} / t$ by tuning $U$ at fixed high detuning $\nu$. In contrast to the result shown in Fig. 3 (a), by decreasing the fraction of closed channel states, as in panels (b) and (c), we find localized insulating phases. This localization can be demonstrated by a divergence in the effective pair mass. In summary, insulating states at non-integer filling are robust even in the presence of closedchannel molecules.

The experimental implications of our work are readily testable, since, fortunately, using Feshbach resonances, current cold atom experiments [2] are able to simulate these attractive Hubbard Hamiltonians. Our principal result is the theoretical observation of superfluid-insulator transitions in the ground state of the attractive Hubbard model away from integer filling. To observe these new phases experimentally one needs to consider average densities $n \approx 1$ and sufficiently large $|U| / t$ of the order of, or larger than, that required for superfluid-Mott insulator transitions in Bose gases [4]. Because experimentally there is an additional background harmonic trapping potential, $n$ can never be precisely specified throughout the lattice [20] and thus it should be possible to find extended regions with non-integer filling factors. The tunable parameters in optical lattice experiments are scattering length and lattice potential depth $V_{0}$. While it is relatively easy to express $t$ in terms of $V_{0}$, the conversion of the on-site attraction $U$ in terms of the scattering length and $V_{0}$ near unitarity is not as straightforward as that in free space [4].

It is clear that the crucial test of the new phase diagram in Fig. 2(b) does not lie in distinguishing whether $T_{c}$ is small or strictly zero. Rather with $T_{c}=0$ one can invoke entropic considerations and deduce that signatures of this new ground state will involve detecting some new form of (bosonic) order. This may be the analogue of a (pair density wave) phase which appears to be present in high temperature superconductors, in the underdoped side of the phase diagram [22].

This work is supported by NSF Grant No. PHY-0555325 and NSF-MRSEC Grant No. DMR-0213745. We thank Jit Kee Chin for helpful conversations.
[1] R. Micnas, J. Ranninger, and S. Robaszkiewicz, Rev. Mod. Phys. 62, 113 (1990).

[2] J. K. Chin, D. E. Miller, Y. Liu, C. Stan, W. Setiawan, C. Sanner, K. Xu, and W. Ketterle, Nature 443, 961 (2006).

[3] Q. J. Chen, J. Stajic, S. N. Tan, and K. Levin, Phys. Rep. 412, 1 (2005); K. Levin and Q. J. Chen, e-print cond-mat/0611104.

[4] M. Greiner, O. Mandel, T. Esslinger, T. Hansch, and I. BLoch, Nature 415, 39 (2002).

[5] A. Sewer, X. Zotos, and H. Beck, Phys. Rev. B 66, 140504(R) (2002).

[6] A. Toschi, P. Barone, M. Capone, and C. Castellani, New Jour. Phys 7, 7 (2005).

[7] P. Nozières and S. Schmitt-Rink, J. Low Temp. Phys. 59, 195 (1985).

[8] Q. J. Chen, I. Kosztin, B. Jankó, and K. Levin, Phys. Rev. B 59, 7083 (1999).

[9] L. M. Duan, Phys. Rev. Lett. 95, 243202 (2005).

[10] L. D. Carr and M. J. Holland, Phys. Rev. A 72, 031604(R) (2005).

[11] F. Zhou, Phys. Rev. B 72, 220501(R) (2005).

[12] H. Zhai and T. L. Ho, e-print, cond-mat/07042957.

[13] H. Heiselberg, C. J. Pethick, H. Smith, and L. Viverit, Phys.
Rev. Lett. 85, 2418 (2000).

[14] E. Burovski, N. Prokof'ev, B. Svistunov, and M. Troyer, Phys. Rev. Lett. 96, 160402 (2006).

[15] A. Bulgac, J. E. Drut, and P. Magierski, Phys. Rev. Lett. 96, 090404 (2006).

[16] E. Burovski, N. Prokof'ev, B. Svistunov, and M. Troyer, Phys. Rev. Lett. 96, 160402 (2006).

[17] Q. J. Chen, K. Levin, and I. Kosztin, Phys. Rev. B 63, 184519 (2001).

[18] J. Stajic, J. N. Milstein, Q. J. Chen, M. L. Chiofalo, M. J. Holland, and K. Levin, Phys. Rev. A 69, 063610 (2004).

[19] Q. J. Chen, I. Kosztin, B. Jankó, and K. Levin, Phys. Rev. Lett. 81, 4708 (1998).

[20] M. Kohl, H. Moritz, T. Stoferle, K. Gunter, and T. Esslinger, Phys. Rev. Lett. 94, 080403 (2005).

[21] In the 2 channel limit as distinguished form the 1 channel case we find that $T_{c}$ at $n=2$ is never strictly zero but the deviations are negligible.

[22] H. D. Chen, O. Vafek, A. Yazdani, and S. C. Zhang, Phys. Rev. Lett. 93, 187002 (2004). 